\title{
A prática de futebol recreativo no Brasil por adultos diabéticos e hipertensos
}

\section{Recreational soccer practice in Brazil for diabetic and hypertense adults}

\author{
Dartel Ferrari Lima ${ }^{1}$, Lohran Anguera Lima², Maria das Graças Anguera ${ }^{3}$, \\ Adelar Aparecido Sampaio ${ }^{4}$, Oldemar Mazzardo Júnior ${ }^{5}$, Olinda do Carmo Luiz ${ }^{6}$
}

\section{Resumo}

\begin{abstract}
O estudo analisou a prática de futebol recreativo de brasileiros diabéticos e hipertensos adultos (18 anos de idade ou mais) e verificou a contribuição dessa modalidade para a obtenção da meta de atividade física recomendada pela Organização Mundial da Saúde. Dados transversais sobre a prática de atividade física foram obtidos do Ministério da Saúde de 2014, em que, cerca de 41.000 adultos foram entrevistados por inquérito telefônico. Aproximadamente 2,4\% dos diabéticos e 3,1\% dos hipertensos relataram o futebol como a principal atividade física recreativa, com predominância de homens de meia-idade (35-44 anos), casados e com nove a 12 anos de escolaridade. A maior parte deles $(80 \%)$ praticavam futebol uma a duas vezes na semana com duração diária de 60 minutos ou mais. Entre os futebolistas recreativos, $80 \%$ dos hipertensos e $20 \%$ dos diabéticos realizavam atividade física suficiente. Os diabéticos necessitam ser informados sobre a importância da regularidade das atividades para maximizar os efeitos da atividade física no controle glicêmico.
\end{abstract}

Palavras-chave: Atividade motora. Diabetes mellitus. Futebol. Hipertensão. Recreação.

1 Doutorado em Ciências pela Faculdade de Medicina da Universidade de São Paulo, São Paulo, São Paulo, Brasil. Professor Permanente do Programa de Pós-Graduação em Educação em Ciências e Educação Matemática da Universidade Estadual do Oeste do Paraná, Marechal Cândido Rondon, Paraná, Brasil. E-mail: dartelferrari07@gmail.com

2 Especialização em Ortopedia e Traumatologia. Residente do Departamento de Ortopedia e Traumatologia da Santa Casa de Misericórdia de São Paulo, São Paulo, São Paulo, Brasil.

3 Doutorado em Ciências (Medicina Preventiva) pela Faculdade de Medicina da Universidade de São Paulo, São Paulo, São Paulo, Brasil. Professora Adjunta da Universidade Estadual do Oeste do Paraná, Marechal Cândido Rondon, Paraná, Brasil.

4 Doutorado em Educação pela Pontifícia Universidade Católica do Rio Grande do Sul, Porto Alegre, Rio Grande do Sul, Brasil. Docente na Universidade Estadual do Oeste do Paraná, Marechal Cândido Rondon, Paraná, Brasil.

5 Doutorado em Desenvolvimento e Aprendizagem Motora pela Universidade de Pittsburgh, Pittsburgh, Pensilvânia, Estados Unidos da América. Professor Adjunto na Universidade Estadual do Oeste do Paraná, Marechal Cândido Rondon, Paraná, Brasil.

6 Doutorado em Ciências (Medicina Preventiva) pela Faculdade de Medicina da Universidade de São Paulo, São Paulo, São Paulo, Brasil. Pesquisadora Científica do Laboratório de Investigação Médica do Hospital das Clínicas da Faculdade de Medicina da Universidade de São Paulo, São Paulo, São Paulo, Brasil. 


\section{Abstract}

The study analyzed the recreational soccer practice of diabetics and hypertensive adults (18 years of age or older) and verified the contribution of this modality to the analyzes of the physical activity goal recommended by the World Health Organization. Cross-sectional data on the practice of physical activity were examined by the Ministry of Health in 2014, which approximately 41,000 adults were interviewed by phone. Approximately $2.4 \%$ of diabetics and $3.1 \%$ practice hypertension related to football as the main recreational physical activity, with a predominance of middle-aged men (35-44 years), married and with 9 to 12 years of schooling. Most of them (80\%) play football once twice a week, with a daily duration of 60 minutes or more. Among recreational footballers, $80 \%$ of hypertensive and $20 \%$ of diabetics perform sufficient physical activity. Diabetics select information about the importance of regular activity to maximize the effects of physical activity without glycemic control.

Keywords: Diabetes mellitus. Hypertension. Motor activity. Recreation. Soccer.

\section{Introdução}

A tendência da taxa de mortalidade é um dos principais indicadores para definir o estado de saúde de uma população. As últimas décadas foram marcadas por uma inversão no perfil global da mortalidade humana. Atualmente, o impacto causado à saúde por doenças crônicas não transmissíveis (DCNT) supera o causado pelas doenças infecciosas. ${ }^{(1)}$ A Organização Mundial da Saúde (OMS) estima que o número total de mortes por DCNT tende aumentar $17 \%$ na próxima década, e a taxa de mortalidade por DCNT será duas vezes maior do que a taxa de mortalidade por doenças infecciosas. ${ }^{(2)}$

De especial importância, a hipertensão arterial e o diabetes, duas das doenças crônicas mais prevalentes na população adulta, despertam preocupação nos profissionais da saúde em todo o mundo. Ambas preocupam por apresentarem, quase sempre, uma fase inicial assintomática. No ano de 2014 , cerca de $8,5 \%$ da população adulta no mundo era composta por diabéticos e essa prevalência resultou em 3,7 milhões de mortes. ${ }^{(3)} \mathrm{O}$ Brasil suporta o quarto o maior número global de diabéticos, com aproximadamente 13,4 milhões de portadores (6,7\% da população). No ano de 2010, aproximadamente 54 mil brasileiros morreram devido ao diabetes ou por suas complicações. ${ }^{(4)}$

A hipertensão arterial sistêmica é responsável por 9,4 milhões de mortes no mundo a cada ano e por $10 \%$ de todo o custo financeiro global para a saúde. Estima-se que em 2025, em todo o mundo, acometerá cerca de 1,6 bilhão de pessoas de ambos os sexos. ${ }^{(5)}$ No Brasil, a hipertensão arterial atinge cerca de $30 \%$ da população adulta, chegando a 5\% nas crianças e adolescentes e a $50 \%$ nas pessoas a partir dos 65 anos de idade. ${ }^{(6)}$ Segundo um estudo global envolvendo 190 países no período de 2001 a 2011, o Brasil apresentou a sexta maior proporção de hipertensos (552:100 mil) com aumento de 13,2 pontos percentuais ao longo da série histórica. ${ }^{(7)}$

Cada vez mais os estudos reforçam a ideia de que a opção por um estilo de vida mais saudável pode interferir de modo positivo na maioria das DCNT. ${ }^{(8)}$ Essa constatação é importante por mostrar que essas doenças apresentam consequências evitáveis. Apesar de a atividade física (AF) regular ser amplamente reconhecida como meio para prevenir ou amenizar as consequências da hipertensão arterial sistêmica e do diabetes, ${ }^{(1-4)}$ o percentual de adultos fisicamente ativos em todo o mundo ainda é reduzido. Globalmente, $16 \%$ da população em países de baixa renda e $37 \%$ em países de alta renda estavam inativos no ano de 2016, sendo adicionado a esses mais um terço da população que não praticava atividade física na quantidade recomendada. ${ }^{(3)}$ No Brasil, cerca de $70 \%$ da população adulta encontramse inativos ou abaixo das recomendações globais de atividade física. ${ }^{(9)}$ 
O monitoramento de informações sobre a atividade física de brasileiros é importante e necessário para estabelecer e acompanhar as ações destinadas aos cuidados de saúde mediados pela promoção da $\mathrm{AF}$, além de subsidiar informações ao público em geral sobre o tipo e a quantidade ideal de AF necessária para obter benefícios substanciais para a saúde, possibilitando às pessoas oportunidades para o autocontrole da promoção e preservação da própria saúde.

A combinação do tipo de AF, da frequência semanal e da duração diária determinam o volume semanal das atividades. O volume semanal, por sua vez, determina a magnitude dos benefícios gerais à saúde. Atualmente (em 2019), há mais de uma recomendação de $\mathrm{AF}$ em vigor. ${ }^{(10)} \mathrm{O}$ Departamento de Saúde dos Estados Unidos, acompanhado pela OMS, recomenda que adultos devem fazer pelo menos 150 minutos de AF de intensidade moderada, ou 75 minutos de intensidade vigorosa por semana, ou qualquer combinação equivalente dos dois. Quando adultos com condições crônicas limitantes de saúde não são capazes de atender às recomendações acima, é sugerido que se envolvam em AF regular de acordo com as habilidades e limitações, evitando, sempre que possível, a inatividade física. ${ }^{(11)}$

A participação no futebol recreativo, aquele praticado no tempo de lazer e com propósito lúdico, está presente no cotidiano dos brasileiros. No entanto, há razoáveis evidências de que diferentes estratos sociais apresentam diferentes níveis de aceitação a determinados espaços destinados à prática das atividades. Ainda, ao relacionar a dimensão temporal do lazer com o futebol, tanto o lazer como o futebol foram afetados pelas constantes transformações dos espaços e dos costumes desenvolvidos ao longo tempo. O futebol recreativo com larga predominância masculina ressalta as relações de domínio e de poder de gênero também manifestadas no esporte, já que essas relações são fenômenos sociais. ${ }^{(9)}$

Atualmente, apresenta-se razoável a quantidade de informações sobre o comportamento da AF de brasileiros adultos. ${ }^{(12-13)}$ Esses estudos têm focado a generalização das atividades, o que parece ser apropriado para o objetivo de proteção à saúde. No entanto, informações de atividades específicas ainda são raras. Assim, este estudo se difere dos demais por analisar um tipo específico de atividade física - o futebol recreativo - praticado por uma parcela específica da população - portadores de diabetes e hipertensos. Desse modo, o estudo analisou o perfil da prática de futebol recreativo em adultos portadores de diabetes e hipertensão arterial residentes nas 26 capitais das unidades federativas do Brasil e do Distrito Federal e verificou como a participação nessa modalidade, isoladamente, contribui para o alcance da meta de AF recomendada pela OMS.

\section{Material e Método}

\section{Amostra e sujeitos}

Foram examinados dados transversais do Sistema de Vigilância de Fatores de Risco e Proteção para Doenças Crônicas não Transmissíveis por Inquérito Telefônico (Vigitel) nas capitais dos estados brasileiros e no Distrito Federal no ano de 2014, cuja metodologia própria pode ser acessada em publicações prévias. ${ }^{(14)}$ Desde 2006, as entrevistas do sistema Vigitel ocorrem anualmente, sendo realizadas por telefone, com pelo menos 2.000 entrevistas com adultos com idade igual ou maior de 18 anos, em cada uma das 26 capitais das unidades federativas do Brasil e do Distrito Federal, cujas moradias apresentam linha telefônica fixa. Pesos pósestratificação são empregados como tentativa de representar as populações com e sem telefone fixo. Em 2014, 40.853 entrevistas foram realizadas. Naquele ano (2014), o Vigitel estabeleceu um tamanho amostral mínimo de 2.000 indivíduos para cada cidade, para estimar, com coeficiente de confiança de $95 \%$ e erro máximo de dois pontos percentuais, a frequência dos principais fatores de risco para as doenças crônicas não 
transmissíveis na população adulta $(\geq 18$ anos de idade). Erros máximos de três pontos percentuais são esperados para as estimativas específicas segundo o sexo, assumindo-se pesos amostrais que proporcionalizaram a diferença entre os sexos. ${ }^{(15)}$

\section{Procedimentos e medidas}

Os participantes foram questionados sobre o principal tipo de exercício ou esporte praticado no tempo livre nos últimos três meses (apenas uma resposta foi aceita) diante uma lista com 16 opções. A frequência semanal e a duração diária das atividades foram, respectivamente, determinadas pelas respostas às questões: "Quantos dias por semana o(a) sr.(a) costuma praticar exercício físico ou esporte?". "No dia em que o(a) sr.(a) pratica exercício ou esporte, quanto tempo dura esta atividade?". Atividade com duração inferior a 10 minutos não foi considerada para efeito de cálculo. ${ }^{(11)}$

A intensidade do esforço foi determinada pelo mesmo parâmetro utilizado pelo Vigitel na edição de 2014. Assim, o futebol recreativo foi classificado como uma atividade de intensidade vigorosa. ${ }^{(15)}$

A frequência dos participantes que referiram diagnóstico médico prévio de hipertensão arterial (sistêmica) e de diabetes foi obtida, respectivamente, conforme a resposta afirmativa dada às questões: "Algum médico já lhe disse que o(a) sr.(a) tem pressão alta?". "Algum médico já lhe disse que o(a) sr.(a) tem diabetes?".

As variáveis sociodemográficas e individuais dos participantes pesquisaram o consumo abusivo de bebida alcoólica (consumo de cinco ou mais doses para homens e de quatro ou mais doses para mulheres em uma única ocasião, por pelo menos uma vez nos últimos 30 dias). Uma dose de bebida alcoólica corresponde a uma lata de cerveja, uma taça de vinho ou uma dose de qualquer bebida destilada. O consumo abaixo da dose abusiva foi considerado como consumo ocasional. Foi considerado fumante o indivíduo que respondeu afirmativamente à questão "O(a) sr.(a) fuma?". O sexo foi categorizado em masculino e feminino; a faixa etária foi agrupada em seis categorias (18-24 anos; 25-34 anos; 35-44 anos; 45-54 anos; 55-64 anos e $\geq 65$ anos de idade); os anos de estudo foram agrupados em três categorias (0-8 anos; 9-11 anos; $\geq 12$ anos); o estado civil foi agrupado em quatro categorias (casado, solteiro, viúvo e separado) e a distribuição das residências atendeu à divisão regional em vigor (Norte, Nordeste, Centro-Oeste, Sudeste e Sul).

A referência para determinar o alcance da meta de AF para hipertensos foi pelo menos 150 minutos de AF de intensidade moderada, ou 75 minutos de intensidade vigorosa por semana, ou qualquer combinação equivalente dos dois. ${ }^{(16)}$ Para os diabéticos, a meta foi de 150 minutos/semana, com atividades aeróbias distribuídas em três dias na semana em dias não consecutivos. ${ }^{(17)}$

\section{Análise estatística}

O peso final atribuído a cada indivíduo entrevistado pelo sistema Vigitel foi denominado de peso pós-estratificação. Essa inferência tende igualar a composição sociodemográfica estimada para a população de adultos com telefone a partir da amostra Vigitel em cada cidade à composição sociodemográfica que se estima para a população adulta total da mesma cidade. Desse modo, os resultados foram apresentados com frequências relativas. O método 'Rake' foi utilizado para estimar a população total de cada capital com base em dados censitários do ano correspondente. As diferenças nas frequências de diabéticos e hipertensos e seus respectivos intervalos de confiança de 95\% foram apresentados segundo o tipo principal de AF praticado, o sexo, a idade, o tempo de escolaridade, o estado civil, o consumo de cigarro e de bebida alcoólica, a concentração regional, a frequência semanal e a duração das atividades e a obtenção da meta de AF recomendada, e foram determinadas pelo teste qui-quadrado $\left(\chi^{2}\right)$ de Pearson. Os resultados com valor de ' $p$ ' menor do que 0,05 foram considerados 
com diferença estatisticamente significante. Para o processamento dos dados e das análises estatísticas utilizou-se o programa $\operatorname{Stata}^{\circledR} 11.0$.

\section{Aspectos éticos}

O projeto Vigitel foi aprovado pela Comissão Nacional de Ética em Pesquisa para Seres Humanos do Ministério da Saúde. O Termo de Consentimento Livre e Esclarecido foi obtido de maneira verbal no momento do contato telefônico com os entrevistados. O banco de dados é público, está disponível na Internet em: <http://svs.aids. gov.br/download/Vigitel/> e não possibilita a identificação dos entrevistados. Desse modo, este estudo está dispensado de submissão ao sistema CEP/CONEP, conforme a Resolução 510/2016 do Conselho Nacional de Saúde, Art. $1^{\circ}$ (Parágrafo Único: não serão registradas nem avaliadas pelo sistema CEP/CONEP). ${ }^{(18)}$

\section{Resultados}

No ano 2014, 40.853 entrevistas foram realizadas com 25.332 mulheres $(62 \%)$ e 15.521 homens (38\%). Desse total, 4.081 entrevistados se declararam diabéticos (10\%) e 12.958 se declararam hipertensos (32\%). Entre os diabéticos, $43 \%$ referiram praticar alguma modalidade de $\mathrm{AF}$ recreativa nos três meses anteriores à entrevista, sendo que somente $2,4 \%$ dos ativos no lazer optaram pelo futebol como a principal AF recreativa. Entre os hipertensos, $45 \%$ referiram praticar alguma modalidade de AF recreativa, sendo que 3,1\% optaram pelo futebol.

A Tabela 1 mostra a frequência de variáveis sociodemográficas e pessoais de adultos ( $\geq 18$ anos de idade) portadores de diabetes ou hipertensão arterial que relataram o futebol recreativo como o tipo principal de AF realizada no tempo livre. Em 2014, o futebol recreativo foi a quinta principal modalidade referida, evidenciando ser uma atividade predominantemente masculina. Somente $1,6 \%$ de mulheres hipertensas optou pelo futebol. Não houve qualquer referência entres as diabéticas. A faixa etária de maior prevalência de futebolistas recreativos foi de 35 a 44 anos de idade, principalmente entre os casados. A proporção de consumidores de bebida alcoólica e de cigarro se contrastou, com elevado percentual de consumidores de bebidas alcoólicas quando comparado aos consumidores de cigarros. Aproximadamente 8,0\% dos diabéticos e 17,0\% dos hipertensos se declararam fumantes, enquanto $29,0 \%$ dos diabéticos e 19,0\% dos hipertensos se declararam consumidores abusivos de bebida alcoólica. As Regiões Norte e Nordeste, com as menores médias de desenvolvimento humano do País, concentraram o maior percentual de praticantes de futebol recreativo, somando, aproximadamente, $60 \%$ de todas as ocorrências.

Tabela 1 - Percentual* de adultos ( $\geq 18$ anos de idade) diabéticos ou hipertensos praticantes de futebol recreativo e o respectivo intervalo de confiança de $95 \%$, de acordo com o sexo, faixa etária, anos de escolaridade, estado civil, consumo de cigarro e álcool e distribuição regional. Vigitel, 2014.

\begin{tabular}{llrrrr}
\hline & & \multicolumn{2}{c}{ DIABÉTICOS } & \multicolumn{2}{c}{ HIPERTENSOS } \\
\hline \multirow{2}{*}{ Sexo } & & $\mathbf{\%}$ & $\mathbf{I C}$ 95\% & \% & IC 95\% \\
\cline { 3 - 6 } & Homens & 100,0 & & 98,4 & $(96,5 ; 99,8)$ \\
Idade (anos) & Mulheres & - & - & 1,6 & $(1,3 ; 2,1) \#$ \\
& $18-24$ & 18,3 & $(16,9 ; 19,9)$ & 16,3 & $(15,2 ; 17,5$ \\
& $25-34$ & 26,7 & $(24,7 ; 28,1)$ & 27,8 & $(26,1 ; 29,2)$ \\
& $35-44$ & 33,3 & $(30,1 ; 35,6)$ & 29,6 & $(27,7 ; 31,5)$
\end{tabular}


Continuação

\begin{tabular}{llrrrl} 
& $45-54$ & 18,3 & $(16,9 ; 19,9)$ & 19,4 & $(17,7-; 22,0)$ \\
Escolaridade & $55-64$ & 3,4 & $(2,7 ; 4,4)$ & 6,5 & $(5,1 ; 7,8) \#$ \\
(anos de estudo) & $\geq 65$ & - & - & 0,4 & $(0,1 ; 0,7)$ \\
\multirow{5}{*}{ Estado civil } & $0-8$ & 20,0 & $(18,8 ; 21,9)$ & 27,8 & $(25,9 ; 29,5)$ \\
& $9-12$ & 46,7 & $(44,2 ; 48,6)$ & 49,5 & $(47,6 ; 52,1)$ \\
& $\geq 12$ & 33,3 & $(30,9 ; 35,6)$ & 22,7 & $(20,6 ; 24,6) \#$ \\
Tabagismo & Solteiro & 38,3 & $(36,7 ; 40,1)$ & 29,3 & $(27,2 ; 32,1)$ \\
& Casado & 56,7 & $(53,6 ; 58,9)$ & 66,4 & $(63,7 ; 69,6)$ \\
Consumo de álcool & Viúvo & - & - & 0,9 & $(0,5-; 1,3)$ \\
& Separado & 5,0 & $(4,1 ; 6,2)$ & 3,4 & $(2,3 ; 4,4)$ \\
& Sim & 8,3 & $(7,1 ; 9,2)$ & 17,3 & $(15,6 ; 19,1) \#$ \\
& Não & 91,7 & $(88,3 ; 94,5)$ & 82,7 & $(79,5 ; 87,1)$ \\
& Abusivo** & 29,1 & $(23,1 ; 35,4)$ & 19,1 & $(13,1 ; 25,4) \#$ \\
& Ocasionalmente & 1,7 & $(0,8 ; 2,2)$ & 1,6 & $(0,5 ; 2,2)$ \\
& Não & 69,2 & $(62,8 ; 76,4)$ & 79,3 & $(76,1 ; 84,9)$ \\
& Norte & 34,1 & $(31,2 ; 36,5)$ & 29,0 & $(26,9 ; 33,1)$ \\
& Nordeste & 27,4 & $(25,7 ; 29,3)$ & 33,1 & $(27,7 ; 36,1)$ \\
& Centro-Oeste & 11,3 & $(10,1 ; 12,9)$ & 16,2 & $(14,4 ; 17,7) \#$ \\
& Sudeste & 15,9 & $(14,2 ; 16,7)$ & 11,7 & $(9,0 ; 12,9) \#$ \\
& Sul & 11,3 & $(9,8 ; 12,8)$ & 10,0 & $(8,6 ; 11,9)$ \\
& & & &
\end{tabular}

Fonte: Autores

* Percentual ponderado para ajustar a distribuição sociodemográfica da amostra Vigitel à distribuição da população adulta de cada cidade projetada para o ano de 2016.

Vigitel: Vigilância de Fatores de Risco e Proteção para Doenças Crônicas por Inquérito Telefônico.

IC 95\%: Intervalo de Confiança de 95\%.

**Cinco doses de bebida destilada consumida em uma única ocasião nos últimos 30 dias da entrevista para homens e quatro doses para mulheres.

\#Diferença estatística significante (teste qui-quadrado de Pearson).

A Tabela 2 mostra o percentual de adultos ( $\geq 18$ anos de idade) portadores de diabetes ou hipertensão arterial segundo a frequência semanal, a duração diária da AF e a obtenção da meta de AF recomendada pela OMS. Aproximadamente $76 \%$ dos diabéticos e hipertensos que têm o futebol como a principal atividade física recreativa praticaram essa atividade entre uma a duas vezes por semana. A duração de cada evento, para cerca de $80 \%$ da amostra foi de 60 minutos ou mais. O volume semanal do futebol recreativo conduziu, aproximadamente, $88 \%$ dos futebolistas hipertensos e $20 \%$ dos futebolistas diabéticos a alcançarem a meta mínima de AF recomendada pela OMS. 
Tabela 2 - Percentual* de adultos ( $\geq 18$ anos de idade) diabéticos ou hipertensos praticantes de futebol recreativo e o respectivo intervalo de confiança de $95 \%$, de acordo com a frequência semanal das atividades, a duração de cada sessão e a obtenção da meta recomendada pela OMS de 2010. Vigitel, 2014.

\begin{tabular}{llrlrl}
\hline & & \multicolumn{2}{c}{ DIABÉ TICOS } & \multicolumn{2}{c}{ HIPERTENSOS } \\
\hline & & $\mathbf{\%}$ & IC 95\% & \% & IC 95\% \\
\cline { 3 - 6 } Frequência (dias/semanal) & Todos os dias & - & - & 2,2 & $(1,3 ; 5,7)$ \\
& 5 a 6 & 7,1 & $(5,8 ; 9,4)$ & 3,3 & $(1,8 ; 4,4) \#$ \\
& 3 a 4 & 16,7 & $(11,1 ; 23,9)$ & 17,6 & $(13,9 ; 21,6)$ \\
& 1 a 2 & 76,2 & $(71,5 ; 85,9)$ & 76,9 & $(72,6 ; 84,1)$ \\
Duração de cada sessão (minutos) & $10-19$ & 0,5 & $(0,01 ; 1,1)$ & 1,0 & $(0,3 ; 1,9) \#$ \\
& $20-29$ & 0,9 & $(0,02 ; 1,9)$ & 2,1 & $(1,3 ; 3,3) \#$ \\
& $30-39$ & 5,6 & $(4,4 ; 6,5)$ & 8,9 & $(7,8 ; 9,6) \#$ \\
& $45-51$ & 6,2 & $(5,1 ; 7,4)$ & 9,3 & $(8,4 ; 10,3) \#$ \\
& $\geq 60$ & 86,7 & $(83,4 ; 89,0)$ & 78,8 & $(75,8 ; 81,1)$ \\
& Sim & 19,7 & $(16,8 ; 23,1)$ & 88,1 & $(83,3 ; 93,6) \#$ \\
\hline
\end{tabular}

Fonte: Autores

* Percentual ponderado para ajustar a distribuição sociodemográfica da amostra Vigitel à distribuição da população adulta de cada cidade projetada para o ano de 2016.

Vigitel: Vigilância de Fatores de Risco e Proteção para Doenças Crônicas por Inquérito Telefônico.

IC 95\%: Intervalo de Confiança de 95\%.

OMS/2010. Recomenda 150 minutos de atividade aeróbia por semana de intensidade moderada, ou 75 minutos por semana de atividade aeróbia de intensidade vigorosa; de preferência, espalhada ao longo da semana. Especificamente para os diabéticos é recomendado o mesmo volume de atividade; no entanto, distribuído em três dias na semana em dias não consecutivos.

\#Diferença estatística significante (teste qui-quadrado de Pearson).

\section{Discussão}

Este estudo descreveu o padrão da $\mathrm{AF}$ de diabéticos e hipertensos com 18 anos ou mais de idade, residentes nas capitais brasileiras, entrevistados por inquérito telefônico e que relataram o futebol como a principal atividade física recreativa. $\mathrm{O}$ futebol foi a quinta preferência entre as modalidades de AF, predominada por homens, com redução de participantes a partir dos 44 anos de idade e encerramento das atividades por volta dos 65 anos de idade. A duração diária das atividades com 60 minutos ou mais foi a principal escolha para a maior parte dos hipertensos (79\%) e dos diabéticos (87\%), enquanto a frequência semanal de uma a duas vezes por semana foi a principal para aproximadamente $80 \%$ da amostra.

O futebol historicamente aparece como um esporte predominantemente masculino, vinculado à virilidade que é característico do referido gênero. Assim, a participação de mulheres é rodeada de preconceitos socioculturais. ${ }^{(9)}$ Mais recentemente foi ampliado o processo da inserção feminina no esporte, integrando pessoas de diferentes origens sociais, culturais, religiosas e raciais. Contudo, pode-se inferir que esse processo de integração ainda não é um caminho finalizado.

Um dos poucos estudos conhecidos e representativo da população brasileira que descreve características sociais e de saúde de adultos praticantes de futebol recreativo no Brasil ocorreu em 2013, realizado por Florindo, ao analisar 54.369 
entrevistas do sistema Vigitel/2006. Naquele estudo, o futebol recreativo foi a principal AF para aproximadamente $10 \%$ da população brasileira. ${ }^{(19)}$ Para o ano de 2014, este percentual diminuiu para 4,4\%. ${ }^{(15)}$ É possível, na comparação direta entre os estudos, verificar que ao longo desse período (oito anos) houve redução superior a 50\% na preferência do futebol como principal AF recreativa.

A redução de parcela da população brasileira para a prática do futebol recreativo parece não implicar em menor taxa de AF de diabéticos e hipertensos, e sim, em uma mudança de escolha do tipo principal de AF. Essa afirmação se sustenta ao verificar a estabilização da frequência de diabéticos e hipertensos ativos no período de $2011^{(20)}$ (42\% e 43\%, respectivamente) para 2014 $\left(45,5 \%\right.$ e $45,0 \%$, respectivamente). ${ }^{(15)}$ Tal cenário de troca de atividade parece razoável à medida que pessoas com maior limitação crônica optem por atividades menos intensas e de menores impactos cardiocirculatório e ortopédico. A troca de uma atividade por outra se apresenta menos preocupante que o cenário de abandono.

$\mathrm{O}$ tipo de $\mathrm{AF}$ e o modo como é praticado (frequência semanal e a duração da atividade) determina o volume semanal da atividade em direção às metas recomendadas. Muito embora algumas diretrizes de AF não fixam a frequência semanal para o público em geral, ${ }^{(10)}$ a necessidade da regularidade parece importante para os diabéticos. A sensibilização da insulina mediada pela $\mathrm{AF}$, a qual garante os efeitos de diminuição da glicemia sérica, é máxima de 12 a 48 horas após a sessão de exercício, voltando progressivamente aos níveis de pré-atividade após 72 horas. ${ }^{(21)}$ Essa plausibilidade biológica é o argumento principal para a orientação de que as atividades de diabéticos possam ser realizadas em dias alternados, com intervalo não maior que 48 horas entre as sessões. A frequência semanal reduzida pode explicar, em parte, porque os resultados de alguns estudos não apontaram melhoria do controle glicêmico de diabéticos menos ativos na mesma proporção que diabéticos mais ativos. ${ }^{(22)}$
Este estudo mostrou que $80 \%$ dos diabéticos apresentaram frequência semanal das atividades entre uma a duas vezes. Em muitos casos, a soma do volume semanal foi suficiente para o alcance da meta; porém, a reduzida frequência semanal, supostamente por não atender aos níveis ideais de controle glicêmico, não se alinha às recomendações de AF específicas para diabéticos. A reduzida frequência semanal observada na prática do futebol pode ser explicada em parte pela associação com outros tipos de exercícios menos vigorosos, pela dificuldade de reunir simultaneamente um grupo de mesmo interesse ou pela menor disponibilidade de espaços para a prática desse esporte, tradicionalmente feita em áreas públicas e em terrenos desocupados, hoje reduzidos nos grandes centros urbanos pela especulação imobiliária.

Para obter efeitos substanciais de saúde mediados pela prática de $\mathrm{AF}$, algumas organizações, como a OMS e o Centro de Controle e Prevenção de Doenças dos Estados Unidos (em inglês: Centers for Disease Control and Prevention - CDC), não fixaram uma determinada frequência semanal em direção à meta recomendada. Essa decisão permite que pessoas alcancem a meta de diversas maneiras. No entanto, a procura da meta em episódios reduzidos (baixa frequência semanal), no Brasil denominado de "atleta de final de semana", pode, devido à concentração do esforço físico, apresentar efeitos adversos a hipertensos e diabéticos. ${ }^{(10)}$ Nesse sentido, parece prudente ressaltar a importância da regularidade das atividades.

A baixa prevalência de tabagismo corrobora os estudos que apontam associação inversa do tabagismo com a $\mathrm{AF}^{(23)}$ sendo possível supor que a prática regular de $\mathrm{AF}$ pode contribuir fortemente para que não fumantes se mantenham afastados do vício do tabaco. Isso pode ser favorável para o não acúmulo de comorbidades causadas pelo uso de cigarro e para o agravamento das doenças de base.

Um fato intrigante dos resultados e que não passou despercebido foi o percentual de consumidores abusivos de bebida alcoólica. 
Cerca de $30 \%$ dos hipertensos e $20 \%$ dos diabéticos relataram o uso abusivo. Para diabéticos do tipo 2, ingerir bebidas alcoólicas abusivamente pode provocar piora da condição de saúde com aumento dos níveis de glicose no sangue e ganho de peso. Isso porque as bebidas alcoólicas são, geralmente, muito calóricas. ${ }^{(24)}$ Para hipertensos, a redução do consumo abusivo de bebidas alcoólicas está associada à redução da pressão arterial de uma forma dependente da dose. ${ }^{(25-26)}$ Identificar pessoas que bebem álcool abusivamente e a implementação de intervenções eficazes para reduzir esse consumo poderia diminuir substancialmente os efeitos de doença do álcool, pressão arterial elevada e do diabetes.

As diretrizes de AF recomendadas pelas organizações de saúde governamentais e não governamentais focam principalmente nos benefícios biológicos às pessoas, mas esses benefícios não constituem a única razão pela qual as pessoas deveriam estar ativas. A AF pode proporcionar diversão, convivência com amigos e familiares, desfrute do meio ambiente, melhora da aparência pessoal e da condição física, entre outras. Nesse sentido, as orientações deveriam encorajar as pessoas a serem fisicamente ativas por toda e qualquer fundamentação que lhes seja significativa. A identificação dos obstáculos para a integração da AF de forma regular no cotidiano e a elaboração de políticas de saúde incentivadoras à prática de $\mathrm{AF}$ poderiam ajudar a diversificar $\mathrm{e}$ massificar as diferentes opções de AF e reduzir as desigualdades de acesso entre os sexos, permitindo às pessoas a proteção contra as DCNT, especialmente aquelas cujos benefícios da AF estão largamente reconhecidos.

Algumas limitações deste estudo devem ser consideradas na interpretação dos resultados. Os dados do Vigitel apresentam apenas a principal modalidade praticada nos últimos três meses, não permitindo capturar outras modalidades praticadas paralelamente. Medidas indiretas de esforço, como as utilizadas pelo sistema Vigitel podem não corresponder fielmente à realidade. Medidas diretas, mais precisas, são caras e logisticamente difíceis em amostra numerosa como deste estudo. Outra limitação se refere ao uso de entrevistas por telefone fixo, já que não considera as pessoas que não os possuem. O uso de pesos pós-estratificados minimiza possíveis diferenças entre a população total e a população do estudo. Por ser realizado mediante entrevistas telefônicas, o Vigitel não pode aferir in loco as doenças relatadas; assim, o número de indivíduos com diagnóstico médico prévio de diabetes e hipertensão pode estar subestimado.

O ponto forte deste estudo foi fornecer informações relevantes, especialmente aos profissionais de saúde e agentes interessados em planejar e promover a AF de diabéticos e hipertensos. A análise do padrão da prática do futebol recreativo e das características dos praticantes reforça a necessidade de ações para incentivar e promover a diversidade de modalidades de AF no cotidiano, de modo a assegurar a continuidade da AF quando o fator idade constituir um forte limitante para a prática de futebol. Assim, recomendam-se aos profissionais de saúde e gestores de políticas públicas voltadas à promoção da AF, explicitar os benefícios da prática do futebol para a saúde dos portadores de DCNT, incentivar a regularidade desta atividade entre os diabéticos, incentivar a aderência feminina para esse esporte e meios para que isso ocorra.

\section{Conclusão}

No Brasil, considerado o "país do futebol”, a prática do futebol recreativo foi a quinta principal opção de AF de lazer na preferência de adultos diabéticos e hipertensos, sendo os futebolistas, predominantemente homens, cerca de $3 \%$ dos diabéticos e hipertensos ativos. Optaram pela prática de futebol recreativo com frequência semanal de uma a duas vezes e duração de 60 minutos por dia. À luz das evidências apresentadas, parece razoável supor que as limitações crônicas influenciam as escolhas de AF menos vigorosas. No entanto, caberia a elaboração e a implementação 
de políticas públicas para a oferta e/ou adequação de espaços de lazer para a prática orientada do futebol recreativo como tratamento não farmacológico, devido à sua reconhecida eficácia em termos de favoráveis adaptações metabólicas, cardiovasculares e musculoesqueléticas.

\section{Referências}

1 World Health Organization Global. Status report on noncommunicable diseases. Geneva: WHO; 2010.

2 Murray CJL, Phil D, Lopez ADL. Measuring the global burden of disease. N Engl J Med. 2013; 369:448-457. doi: 10.1056/NEJMra1201534.

3 World Health Organization. Global report on diabetes. Geneva: WHO; 2016.

4 Ministério da Saúde (BR). Estratégias para o cuidado da pessoa com doença crônica: diabetes mellitus. Brasília (DF): Ministério da Saúde; 2013. (Cadernos de atenção básica, n. 36).

5 World Health Organization (WHO). A global brief on hypertension: silent killer, global public health crisis [Internet]. 2013 [cited 2018 Apr 21]. Available from: http://publichealthwell.ie/ node/466541

6 Secretaria de Estado da Saúde do Paraná (PR). Linha guia de hipertensão arterial / SAS. 2. ed. Curitiba: SESA; 2018.

7 Mozaffarian D, Benjamin EJ, Go AS, Arnett DK, Blaha M, Cushman M, et al. Heart disease and stroke statistics - 2015 update: a report from the American Heart Association. Circulation. 2015;131(4):29-322. doi: 10.1161/ CIR.0000000000000152.

8 Booth FW, Roberts CK, Thyfault JP, Ruegsegger GN, Toedebusch RG. Role of inactivity in chronic diseases: evolutionary insight and pathophysiological mechanisms. Rev Physiol. 2017; 97:1351-1402. doi: doi.org/10.1152/ physrev.00019.2016.
9 Lima DF, Piovani VGS, Lima LA. Recreational soccer practice among adults, in Brazilian capitals, 2011-2015. Epidemiol. Serv. Saude. 2018;27(2):e2017284. doi: org/10.5123/ s1679-49742018000200013.

10 Lima DF, Levy RB, Luiz OC. Recomendações para atividade física e saúde: consensos, controvérsias e ambiguidades. Rev Panam Salud Publica. 2014;36(3):164-70.

11 Department of Health and Human Services (US). Physical Activity Guidelines for Americans, 2nd edition. Washington, DC: U.S. Department of Health and Human Services; 2018.

12 Cruz MS, Bernal RGI, Claro RM. Tendência da prática de atividade física no lazer entre adultos no Brasil (2006-2016). Cad. Saúde Pública. 2018;34(10):e00114817. doi: 10.1590/0102-311X00114817.

13 Hallal PC, KnuthI AG, Reis RS, Rombaldi AJ, Malta DC, Iser BPM, et al. Tendências temporais de atividade física no Brasil (20062009). Rev. bras. Epidemiol. [Internet]. 2011 [citado 2018 jan 04];14(1):53-60. Disponível em: http://www.scielo.br/ scielo.php?script $=$ sci_arttext\&pid $=\mathrm{S} 1415$ $790 \mathrm{X} 2011000500006 \& \operatorname{lng}=\mathrm{en} \& \mathrm{nrm}=$ iso. doi:org/10.1590/S1415-790X2011000500006

14 Ministério da Saúde(BR). Vigitel - Brasil 2017: Vigilância de Fatores de Risco e Proteção para Doenças Crônicas por Inquérito Telefônico. Brasília: Ministério da Saúde; 2018.

15 Ministério da Saúde (BR). Vigitel - Brasil 2014: Vigilância de Fatores de Risco e Proteção para Doenças Crônicas por Inquérito Telefônico. Brasília: Ministério da Saúde; 2015.

16 World Health Organization (WHO). Global recommendations on physical activity for health. Genebra: (WHO); 2010.

17 Organización Mundial de la Salud (OMS). Informe mundial sobre la diabetes. Ginebra: OMS; 2016. 
18 Conselho Nacional da Saúde (BR). Resolução 510, de 7 de abril de 2016. [Internet]. 2016 [citado 2018 maio 24]. Disponível em: http:// conselho.saude.gov.br/resolucoes/2016/ Reso510.pdf

19 Florindo AA, Farias Junior JC, Wells JCK, Hallal PC. Epidemiology of recreational football in Brazil: prevalence and association with risk factors for chronic diseases and self-rated health in adults. Motriz. 2013;19 (1):224-230.

20 Ministério da Saúde (BR). Vigitel - Brasil 2011: Vigilância de fatores de risco e proteção para doenças crônicas por inquérito telefônico. Brasília: Ministério da Saúde; 2012.

21 Bird SR, Hawley JA. Update on the effects of physical activity on insulin sensitivity in humans. 2017 BMJ Open Sport Exerc Med. 2017;2(1):1-27. doi: 10.1136/ bmjsem-2016-000143.

22 Cartee GD. Roles of TBC1D1 and TBC1D4 in insulin- and exercise-stimulated glucose transport of skeletal muscle. Diabetologia. 2015;58:19-30. doi: 10.1007/s00125-0143395-5.

23 Australian Institute of Health and Welfare (AU). Physical activity across the life stages. [Internet]. 2018 [cited 2019 Jan 16]. Available from: https://www.aihw.gov.au/getmedia/ c249ef97-e219-44df-a8bd-f5e50d04064c/ aihw-phe-225.pdf.aspx?inline=true

24 Skovenborg Erik. Shift work and diabetes: alcohol consumption as a risk fator. BMJ. 2019; 364:177. doi: https://doi.org/10.1136/bmj.1177

25 Aladin A, Chevli,P, Ahmad MI, Rasool S, Herrington D. Alcohol consumption and risk of hypertension. J. Am. Coll. Cardiol. 2019;73(9):12. doi: $10.1016 / \mathrm{S} 0735$ 1097(19)33774-X.

26 Roerecke M, Kaczorowski J, Tobe SW, Gmel G, Hasan OSM, Rehm J. The eff ect of a reduction in alcohol consumption on blood pressure: a systematic review and meta-analysis. The Lancet Public Health. 2017;2(2):e108-e120. doi: 10.1016/S2468-2667(17)30003-8. 
Lima, D. F. et al. 